Cahiers
Recherche
surles Droits Fondamentaux
Cahiers de la recherche sur les droits fondamentaux

4 | 2005

Quel avenir pour la laïcité cent ans après la loi de 1905 ?

\title{
Laïcité dans le service public de l'éducation
}

\section{Claude Durand-Prinborgne}

\section{(2) OpenEdition}

Journals

Édition électronique

URL : https://journals.openedition.org/crdf/7253

DOI : $10.4000 /$ crdf.7253

ISSN : 2264-1246

Éditeur

Presses universitaires de Caen

Édition imprimée

Date de publication : 1 décembre 2005

Pagination : 23-32

ISBN : 2-84133-250-0

ISSN : $1634-8842$

Référence électronique

Claude Durand-Prinborgne, "Laïcité dans le service public de l'éducation », Cahiers de la recherche sur les droits fondamentaux [En ligne], 4 | 2005, mis en ligne le 15 décembre 2020, consulté le 14 novembre 2022. URL : http://journals.openedition.org/crdf/7253; DOI : https://doi.org/10.4000/crdf.7253 


\title{
Laïcité dans le service public de l'éducation
}

\author{
Claude DURAND-PRINBORGNE \\ Professeur honoraire de droit public
}

\begin{abstract}
I. Laïcité et organisation du service
A. Le respect des croyances par l'enseignement

B. Respect des croyances : liberté des cultes et droit de recevoir une instruction religieuse

C. Le respect des croyances et les interdits alimentaires
\end{abstract}

II. Laïcité et moyens de service

A. Les personnels

B. Le régime des locaux

III. Laïcité et droits et obligations des usagers
A. Absences pour motifs religieux
B. Manifestations d'appartenance
C. Libertés publiques des élèves et étudiants et manifestations religieuses

IV. Laïcité et obligation hors service public. L'imposition par voie contractuelle d'obligations de laïcité

Dans un Code de l'éducation dont la seule partie législative comprend près de mille articles un chapitre est consacré à la laïcité, il ne comprend que cinq articles dont un pour les enseignements supérieurs. Deux autres articles concernant la laïcité sont inclus dans d'autres parties du texte : un sous le titre « Droits et obligations des élèves » (C. éduc. art. 511-2) et un pour les étudiants (C. éduc. art. L. 811-1). C'est peu. Le dispositif législatif concernant la laïcité dans le service public de l'éducation ${ }^{1}$ ne gagne guère en volume si l'on ajoute l'article 2 de la loi du 9 décembre 1905 concernant la séparation des Églises et de l'État.
Mais on ne juge pas l'importance d'une question au seul nombre des brèves dispositions législatives qui lui sont consacrées. Il suffit d'évoquer pour s'en convaincre l'importance actuelle des débats politiques et sociaux sur l'application du principe de laïcité dans le service public retenu. Il suffit, également, de penser à l'importance, tant en volume qu'en portée, d'une jurisprudence administrative et, conséquence, de la littérature juridique sur la question sous forme de nombreux articles, chroniques et notes.

Cette importance conduit d'ailleurs à s'interroger sur l'intérêt scientifique réel de la présentation d'une synthèse

1. On ne fera pas de la définition de ce qu'il faut entendre par service public de l'éducation un premier objet de recherches, bien que cela puisse se concevoir. La question se pose simplement en préalable. Les textes retiennent des définitions de services publics en fonction de justifications politiques ou de raisons techniques ou des besoins de l'instant. Il existe ainsi un service public de l'enseignement supérieur (C. éduc. art. L. 111-5 ancien art. 3 de la loi Savary $n^{\circ} 84-52$ du 26 janv. 1981). Il existe un service public de l'enseignement agricole. Dans la jurisprudence certains arrêts ont retenu un service public de l'enseignement maternel et élémentaire (CE, 24 novembre 1982, CFDT, AJDA, 1983.155-CE, 16 avril 1986, CFDT, Rec. p. 103) ou de l'enseignement public du second degré (CE, sec., 14 novembre 1975, Ministre de l'Éducation c. $\mathrm{M}^{\mathrm{me}}$ Simon et syndicat national des lycées et collèges, Rec. p. 569). Nous retenons ici ce qui relève classiquement de l’Éducation nationale, principalement les enseignements scolaires et, le cas échéant, les enseignements supérieurs. Précisons simplement que les solutions qui concernent la laïcité sont applicables à l’enseignement agricole. 
sur la laïcité dans le système éducatif français. Plusieurs raisons nous paraissent justifier l'entreprise.

La première raison est que la somme des solutions contentieuses intervenues sur certaines situations telles l'absentéisme scolaire pour raisons religieuses ou le port de signes d'appartenance, par exemple, fait un peu écran. Elle privilégie, par définition, à la fois l'instant et un objet parce qu'il n'est pas de la responsabilité du juge d'élargir les faits d'une espèce, questionnement concret, à un examen d'ensemble de la laïcité. Il n'y a guère de textes et de contentieux sur certains comportements de prosélytismes dénoncés par la conférence des présidents d'universités, ou sur le grave problème de la scolarisation par des mouvements sectaires.

La seconde raison est de s'interroger en droit sur une recherche qu'historiens et sociologues effectuent pour leur part: quelle évolution peut-on constater depuis la fin du $\mathrm{XIX}^{\mathrm{e}}$ siècle, période de détermination des principes qui commandent en la matière hors du banal constat de la diversification des religions? Permanence des questions et des réponses? Mutation?

La troisième raison est qu'il est intéressant de se poser une question, que certains défenseurs de la laïcité refusent de se poser sans doute parce qu'elle détermine partiellement le champ de celle-ci, donc son importance politique réelle, qu'il n'est pourtant nullement question de remettre en cause au plan constitutionnel et dans la conception française de la laïcité : le droit de la laïcité est-il ici simple application d'un droit commun de la laïcité dans les services publics, a-t-il une spécificité, est-il totalement original?

Nous tentons de répondre aux trois questionnements qui sous-tendent nos précédentes justifications de l'entreprise en retenant successivement d'abord l'organisation du service public (I) puis le régime des moyens du service (II) puis la situation des usagers (III). Il nous semble intéressant d'ajouter des développements sur des situations dans lesquelles des obligations de service public sont imposées à des activités extérieures à celui-ci (IV).

\section{Laïcité et organisation du service}

Pour des raisons à la fois historiques - l'École de la République berceau de la laïcité - et politiques - la place constitutionnelle de la laïcité et sa fonction en France les solutions sont ici anciennes, acquises, solides. Le renouvellement des problèmes dans la société existe. Il tient au multiconfessionnalisme actuel ou - il ne nous appartient pas d'en juger la véracité - au renouveau des religiosités. Ceci n'induit pas de bouleversements profonds du droit applicable.

L'organisation du service public était hier affaire exclusivement d'État. Elle le reste largement: «l'éducation est un service public national dont l'organisation et le fonctionnement sont assurés par l'État, sous réserve des compétences attribuées... aux collectivités territoriales » (C. éduc. art. L. 211-1, rédaction de l'art. 75 de la loi n 2004-
809 du 13 août 2004). C'est donc dans des dispositions nationales et dans leurs interprétations qu'il faut rechercher les solutions applicables. Mais il faut préciser que l'État impose le respect de celles-ci aux organismes personnalisés qui relèvent de lui, dans le cadre de l'autonomie légale dont ils jouissent, les universités et les établissements publics locaux d'enseignement, par le biais d'obligations formulées par la loi et les dispositions réglementaires, avec des régimes différents pour celle-là et ceux-ci. Dans les collèges et les lycées, par exemple, le respect du principe de laïcité s'impose tant aux décisions que peuvent prendre les autorités administratives responsables (chef d'établissement, conseil d'administration) que pour la rédaction du règlement intérieur de l'établissement (C. éduc. art. L. 421-4).

Deux grands principes dominent l'action du service public: celui du respect des croyances dans l'enseignement et celui du droit des élèves à l'accès tant à la pratique du culte qu'à l'instruction religieuse. Les solutions du siècle dernier se vérifient mais avec une évolution et une situation nouvelle pour la pratique du culte.

\section{A. Le respect des croyances par l'enseignement}

Le principe d'égal respect de toutes les croyances est formulé par la Constitution et par la loi. Selon le Code de l'éducation, article L. 141-2, « suivant les principes définis dans la Constitution, l'État assure aux enfants et aux adolescents dans les établissements publics d'enseignement la possibilité de recevoir un enseignement... dans un égal respect de toutes les croyances ». Le texte est celui de l'article I de la loi Debré n ${ }^{\circ}$ 59-1557 du 31 décembre 1959. Le principe se retrouve pour l'enseignement supérieur (C. éduc. art. L. 141-6). L'obligation exprimée découle de textes antérieurs. Elle se fonde sur l'alinéa 13 du préambule de la Constitution du 27 octobre 1946 confirmé par celui de la Constitution du 4 octobre 1958 avec la qualification de l'enseignement public : il est laïque. Mais l'obligation procède de la législation dite Ferry: suppression par la loi du 22 mars 1882 de l'instruction morale et religieuse prévue par l'article 23 de la loi du 15 mars 1850, substitution d'une instruction morale et civique, renvoi de tout enseignement religieux hors des édifices scolaires par l'article 2 de la loi (C. éduc. art. L. 141-3) et en dehors des heures de classe pour les écoles publiques (C. éduc. art. L. 141-4, art. 30 de la loi du 9 décembre 1905).

Le problème du respect des croyances par l'enseignement se pose toujours, et il ne peut en aller autrement, au double plan des contenus de l'enseignement, des programmes, et des comportements des maîtres qui dispensent l'enseignement. Ce dernier aspect sera retenu lors de l'examen du régime des moyens du service public.

Selon la formule de la circulaire Jospin du 12 décembre 1989 «l'école publique ne privilégie aucune doctrine. Elle ne s'interdit l'étude d'aucun champ du savoir... L'école publique respecte de façon absolue la liberté de conscience des élèves » ${ }^{2}$. Le texte n'est plus partie de notre 
droit positif mais il méritait d'être cité car il traduisait le droit antérieur et il garde son intérêt car c'est aussi le principe général qui domine les solutions juridiques. La neutralité des programmes a été évoquée de façon incidente par le juge administratif (CE, 2 novembre 1992, MM. Kherouaa et Balo: Rec. p. 389, AJDA, 1992, p. 790 sq.). Elle l'a été directement à propos de l'éducation sexuelle et de la formation à la prévention du sida qui ne portent pas atteinte aux conviction religieuses et philosophiques des élèves et de leurs parents (CE, 18 octobre 2000, Association promouvoir et autres : Rec. p. 425). En réalité le contentieux sur le contenu des programmes est rare et parfois ancien $^{3}$. Les contestations sont plus fréquentes en fait. Elles ne portent pas, d'ailleurs, sur les programmes mais sur les mises en œuvre de ceux-ci dans les manuels scolaires. En l'absence de toute censure préalable de ceuxci l'État est hors de cause. Ceci est dit dans des réponses ministérielles à des questions écrites de parlementaires. L'affaire de l'atteinte aux convictions religieuses par tel ou tel manuel concerne l'auteur et l'éditeur, qui peut le retirer, ce qui se voit parfois.

Les règles qui précèdent ne concernent pas pour des raisons historiques les départements du Bas-Rhin, du Haut-Rhin et de la Moselle. Dans ceux-ci l'enseignement religieux est obligatoire pour les cultes reconnus ${ }^{4}$ selon des modalités différentes dans les écoles confessionnelles, d'une part, interconfessionnelles, d'autre part (TA Strasbourg, 15 mai 1979, M $M^{\text {elle }}$ Monnier et autres c. Éducation nationale, inédit). Le juge a considéré que l'enseignement religieux s'imposait en enseignement technique (CE, 23 mai 1958, Ministre de l'Éducation nationale c. sieur Weber: Rec. p. 293).

Le problème de la place qu'il convient de faire au fait religieux dans l'enseignement est depuis quelques années au cour de débats pour l'ensemble du service public ${ }^{5}$. Il n'est pas question d'introduire un enseignement religieux que la «neutralité d'abstention », que dicte le respect des croyances, interdit. Le fait religieux a été depuis longtemps déjà évoqué dans l'enseignement comme fait d'histoire, fait social, fait de civilisation. Ceci n'a jamais fait problème pour la laïcité. La question actuelle est celle de l'introduction dans les programmes d'une information sur les religions. «L'inculture indifférente n'est pas une garantie de tolérance si elle alimente un espèce d'esprit moi pour tout ce qu'elle ignore, ou si elle favorise la peur de celui qui, en face, affirme hautement sa croyance ${ }^{6}$. La question de la place du fait religieux est sans doute soulevée sur demande constante et forte des grandes religions présentes en France. Elle l'est largement du côté gouvernemental pour des raisons de développement d'un esprit de tolérance fondé sur une compréhension de l'autre. Le Ministère a été à l'origine de divers rapports sur la prise en compte du fait religieux ${ }^{7}$ et l'exposé des motifs de ce qui sera la loi du 15 mars 2004 (infra III) précise qu' « il ne s'agit pas non plus de faire de l'école un lieu d'uniformité et d'anonymat qui ignorerait le fait religieux». La question de la détermination du contenu de cet enseignement, dont le principe ne paraît pas faire problème véritablement, y compris dans des milieux dits laïques, est actuellement en examen. Elle est délicate. Exemple de difficulté : bien que cela n'ait pas donné lieu à un contentieux, dont le succès paraît d'ailleurs bien inconcevable, certains parents, certains élèves parfois, de milieux créationnistes (chrétiens ou musulmans), contesteraient actuellement la présentation des thèses révisionnistes de Darwin.

La loi affirme le caractère laïque du service public de l'enseignement supérieur en des termes dénués de toute équivoque : «laïque et indépendant de toute emprise... religieuse... il tend à l'objectivité du savoir, il respecte la diversité des opinions ", dispose l'article 3 alinéa $1^{\text {er }}$ de la loi no 84-52 du 26 janvier 1984 (C. éduc. art. L. 141-6). Mais les solutions ne s'inscrivent pas dans une conception d'une laïcité « neutralité d'ignorance » ou «neutralité d'abstention » : selon les conclusions du commissaire du gouvernement Helbronner toujours actuelles depuis 1912 «la nature de l'enseignement donné, le caractère des personnes auxquelles il s'adresse, dispense en principe l'État de prendre... la responsabilité des doctrines qui y sont enseignées. Les auditeurs sont en âge de juger » (concl. sous CE, 10 mai 1912, abbé Bouteyre, Rec. p. 553, RDP, 1912, p. 453) et «par leur nature même les fonctions d'enseignement et de recherche non seulement permettent mais demandent, dans l'intérêt du service, que la libre expression et l'indépendance des personnels soient garanties... » (CC, 20 janvier 1984 déc. 83-185 DC : AJDA, 1984, p. 163).

\section{B. Respect des croyances : liberté des cultes et droit de recevoir une instruction religieuse}

«L'État prend toutes dispositions utiles pour assurer aux élèves de l'enseignement public la liberté des cultes et de l'instruction religieuse ", disposait l'article $1^{\text {er }}$ de la loi Debré du 31 décembre 1959 repris par l'article L. 141-2 du Code de l'éducation. Ce texte réaffirme des solutions antérieures, l'une de l'article 2 de la loi du 28 mars 1882, l'autre de la loi du 9 décembre 1905 même si c'est pour dire que l'enseignement religieux sera dispensé hors des établissements scolaires et hors des horaires scolaires (C. éduc. art. L. 141-4) qui organisent le droit de l'élève à recevoir une instruction religieuse.

Le dispositif qui prévoit qu'un jour est affecté dans la semaine scolaire à une possible instruction religieuse est longtemps resté sans faire problème. Des difficultés avec

3. J.-M. Woehling, « Matières régies par le droit public alsacien-mosellan », J. Cl. Adm., fasc. 122-2, $\mathrm{n}^{\circ} 66$.

4. Il a porté sur des question politiques: CE, 20 janvier 1911, Porteret: Rec. p. 68 sq. concl. M. Pichat; CE, 14 janvier 1916, association des pères de famille de Gamache-les-Bains, Rec. p. 30.

5. Voir notre article «La religion au lycée», Rev. Savoir, 1991, p. 1 sq.

6. La formule est de Danièle Hervieu-Léger dans La Religion au lycée, Paris, Cerf, 1990, p. 15.

7. Rapport du recteur Joutard en 1989, rapport R. Debray en mars 2002. Voir R. Debray, L'Enseignement du fait religieux dans l'école lä̈que, Paris, Odile Jacob - SCEREN / CNDP, 2003; J. Willaine, «École et religion : une nouvelle donne », Revue française de pédagogie, 1998, p. 820. 
la hiérarchie catholique ne sont apparues qu'un peu plus d'un siècle après que les règles aient été formulées lors des débuts de l'aménagement de la semaine scolaire qui conduisait dans certaines écoles à concentrer les enseignements sur quatre jours et demi en supprimant ceux du samedi matin déplacés au mercredi... jour de catéchèse pour la religion catholique. Ceci a donné naissance à un contentieux dont les moyens d'annulation ont porté en réalité sur la compétence de l'auteur de la décision ou la procédure d'adoption de celle-ci (TA Poitiers, 25 mai 1988, Monseigneur Rol évêque d'Angoulême c. ministre d'État ministre de l'Éducation nationale : RFDA, 1988, p. 676, avec notre note «L'école, le temps des loisirs et le temps de Dieu », D., 1989, JP, p. 603, note Clapié « La place de l'enseignement religieux dans la semaine scolaire: dernier avatar de la guerre scolaire?» - TA, Orléans, 9 juin 1988, archevêque de Bourges: D., 1989, JP, p. 603, note Clapié préc. - CE, 27 juillet 1990, association pour une nouvelle organisation du temps scolaire: Les Petites Affiches, 18 février 1991, $\mathrm{n}^{\circ}$ 21, p. 18, note J. Fialaire « Rythmes scolaires et exercice du droit à l'enseignement religieux»). Le Conseil d'État a jugé qu'il appartenait au ministre de déterminer par arrêté le jour d'interruption (CE, 27 juillet 1990, préc.). Ce contentieux a été un moment dans le temps alors que l'on sait qu'actuellement un tiers des élèves des écoles maternelles et primaires publiques travaillent dans le cadre d'un aménagement de la semaine scolaire. La lecture des requêtes qui ont justifié les décisions qui précèdent ne permet pas de savoir si des considérations politiques de l'heure auraient pu alors motiver véritablement les recours de représentants de l'Église catholique, motivations qui auraient disparu avec un changement des relations entre l'Église catholique et le Ministère. Il est permis de le supposer compte tenu de la période en cause. La catéchèse a été modifiée. Le ministre a déclaré en août 2005 qu'il n'entendait pas généraliser la semaine aménagée et a renvoyé la décision au plan local avec l'accord des élus, des parents, des enseignants sans aucune référence à une consultation d'autorités religieuses.

Une seconde disposition organise la possibilité de recevoir une instruction religieuse et d'avoir accès au culte : celle de l'article 2 de la loi du 9 décembre 1905 portant séparation des Églises et de l'État qui, après une interdiction de principe du financement public pour l'exercice des cultes, dispose que " pourront toutefois être inscrites auxdits budgets les dépenses relatives à des services d'aumôneries et destinées à assurer le libre exercice des cultes dans les établissements publics tels lycées, collèges, écoles ». Une circulaire du 30 juillet 1946 avait supprimé cette possibilité. Elle a été annulée (CE, $1^{\text {er }}$ avril 1949, abbé Chaveneau: D., 1949, p. 531 ; Rec. p. 161 - CE, 28 janvier 1955, association professionnelle des aumôneries de l'enseignement public: Rec. p. 51). La matière longtemps traitée, de 1905 à 1960, par de simples circulaires a été reprise par voie réglementaire. Elle a suscité un temps des études, expressions parfois d'incertitudes ${ }^{8}$. La question est traitée par les articles R. 141-2 à R. 141-7 du Code de l'éducation ${ }^{9}$. Nous n'en reprenons pas ici les dispositions. Le Conseil d'État a jugé que l'octroi d'une subvention publique par voie d'offres de concours pour la construction et l'entretien d'un pavillon cultuel à l'intérieur d'une cité scolaire était légal (CE, 7 mars 1969, ville de Lille : D., 1969, JP, p. 275 concl. Guillaume; Gaz. Pal., 1962.2, p. 162; RDP, 1969, p. 1170).

Les auditions durant les travaux de la commission Debré sur « la laïcité à l'école : un principe républicain à réaffirmer ${ }^{10}$ ont évoqué le sujet des aumôneries. Il en a été ainsi également pour la commission Stasi. L'orientation politique est de favoriser le développement des aumôneries notamment pour contribuer dans le système scolaire à une meilleure intégration de l'islam dans la société.

Le traitement ancien et classique du problème de l'accès d'élèves - et d'étudiants - à l'instruction religieuse et au culte s'est depuis une dizaine d'années avéré insuffisant et incomplet. La question s'est posée de certaines exigences telles la dispense d'enseignement pour raison religieuse (infra III) ou celle de respect d'interdits alimentaires.

\section{Le respect des croyances et les interdits alimentaires}

L'exigence du respect par la restauration scolaire d'interdits alimentaires est récente. Dans l'enseignement primaire la restauration scolaire ne relève pas de l'Éducation nationale mais de cantines scolaires gérées en régie par les communes, ou concédées ou gérées par des associations. Dans certains cas des repas avec nourriture hallal ou kascher sont assurés par les restaurants scolaires. Elle ne l'est pas partout. Certaines municipalités ont pris prétexte du surcoût de fonctionnement pour cesser de prendre en compte les interdits alimentaires (TA Marseille, 26 novembre 1996, $\mathrm{M}^{\mathrm{me}}$ Zitoussi, Ghribi et autres c. commune de Marignane - CE, ord. 25 octobre 2002, $\mathrm{M}^{\mathrm{me}}$ Renault). Le ministère de l'Éducation nationale préconise par circulaire la prise en compte des coutumes alimentaires familiales notamment pour les enfants d'origine étrangère... ce qui permet de prendre en compte les interdits alimentaires. Ceci concerne les services de bouche des établissements publics locaux d'enseignement ayant des internats ou des demi-pensions.

\section{Laïcité et moyens de service}

Les solutions sont ici acquises et solides mais, d'une part, certaines situations de fait qui conduisent aux rappels de celles-ci sont nouvelles et, d'autre part, le particularisme du service public en cause est souligné dans un

8. J. Rivero, «Les aumôneries de l'enseignement public », D., 1960, chron. p. 79 ; A. Texier, «Les aumôneries de l'enseignement secondaire : incertitudes

d'une institution", RDP, 1984, p. 105 sq.

9. Commentaires in C. Durand-Prinborgne et A. Legrand, Code de l'éducation, LITEC, Juris-code, éd. $2005-2006$ (à paraître).

10. Assemblée nationale, Documents d'information, rapport $\mathrm{n}^{\circ} 1275,2$ tomes, décembre 2003. 
contexte général de rappel des obligations qui incombent aux fonctionnaires et agents publics au titre de la laïcité. La matière est dominée par l'exigence d'une neutralité du service public.

\section{A. Les personnels}

Nous ne reprendrons pas, cela serait inutile, l'ensemble des solutions de notre droit, bien connues, sur le recrutement et les obligations en cours de carrière des personnels. Mais il nous semble intéressant de relever certaines solutions intervenues depuis une vingtaine d'années.

Pour le recrutement des personnels l'article 17 de la loi Goblet du 30 octobre 1886 dispose que « dans les écoles publiques de tout ordre l'enseignement est exclusivement confié à un personnel laïque ». La règle en dehors de sa finalité de sécularisation de l'enseignement est fondée sur le souci de donner aux parents une garantie de respect de toutes les croyances, qui s'ajoute à la gestion des programmes. Elle se retrouve à l'article L. 141-5 du Code de l'éducation avec une modification de libellé qui prend en compte l'évolution de l'organisation du service: «dans les établissements du premier degré public» remplace « dans les écoles publiques de tout ordre». En l'absence d'une règle semblable c'est le Conseil d'État qui, en 1912, confirme la légalité d'un refus administratif d'admettre un prêtre à passer un concours de recrutement (CE, 10 mai 1912, abbé Bouteyre: S, 1912.3, p. 145 concl. Helbronner, note M. Hauriou). Une décision contraire d'un tribunal administratif a jugé un tel refus illégal (TA Paris, 7 juillet 1970, Spagnol, Rec. p. 851). Elle est restée isolée. Des décisions plus récentes ont confirmé, au contraire, l'exigence au moment du recrutement de garanties d'intégration dans un personnel laïque donc l'absence de toute manifestation d'appartenance religieuse ou d'appartenance au clergé (TA Versailles, 14 mai 1992, $\mathrm{M}^{\text {lle }}$ Brazza: Rev. Savoir, 1992, p. 549, non-renouvellement d'une maîtresse de demi-pension pour port du foulard islamique Pour le même motif: refus d'admission en Institut universitaire de formation des maîtres, TA Marseille, 20 octobre 2000, Mlle Montailler c. IUFM de l'académie d'Aix-Marseille). Aucune exigence semblable n'existe pour le personnel de l'enseignement supérieur (cf. concl. Helbronner préc.).

Le principe de laïcité impose aux fonctionnaires et agents du service en cours de carrière ${ }^{11}$ une obligation de réserve. Initialement l'exigence en est fondée sur la volonté de ne pas porter atteinte aux convictions religieuses des familles. La justification par le souci de protéger l'élève vulnérable contre l'abus d'autorité est également constamment avancée ${ }^{12}$. À cela s'ajoute, ce qui est le droit commun des services publics, l'exigence de neutralité religieuse des personnels dans le cadre du principe général de neutralité des services, condition sine qua non du principe d'égalité d'accès et de traitement. La lettre de Jules Ferry aux instituteurs du 17 novembre 1883 est ici souvent citée bien qu'elle concerne en réalité la morale. Ce qui est interdit c'est le prosélytisme, la propagande, la critique partiale et excessive, l'étude ou la citation de textes dont la teneur est incompatible avec la liberté de conscience des élèves. C'est ce que pose la jurisprudence ancienne (TC, 2 juin 1908, Girodet c. Morizot: S, 1908.3, p. 81, note Hariou) et ce qui est confirmé plus récemment (TA Montpellier, 14 avril 1992, Bellatone; TA Paris, 26 juillet 1995, M. et $\mathrm{M}^{\mathrm{me}}$ Noueddene; TA Paris, 25 novembre 1995, M. et M. Benhabib). Longtemps l'exigence de respect de la neutralité religieuse a été imposée aux seuls enseignants. Saisi pour avis par un tribunal administratif le Conseil d'État a estimé que l'obligation de réserve incombait également au personnel non enseignant, en l'espèce le personnel d'éducation (CE, 3 mai 2000, M ${ }^{\text {lle }}$ Martaux: AJDA, 2000, p. 673). La justification par l'application du droit commun des services publics est logique, celle tirée du respect des convictions des parents également mais au surplus d'un point de vue plus éducatif il faut souligner le fait que ces personnels sont membres de la communauté éducative comme les enseignants (C. éduc. art. L. 111-3) et que leur participation aux responsabilités éducatives, notamment de vie scolaire, est de plus en plus soulignée et voulue. Le problème de l'application d'une même obligation de réserve aux parents d'élèves collaborateurs bénévoles du service public lorsqu'ils participent à l'encadrement d'activités éducatives telles des sorties scolaires est posé depuis un certain temps. Les décisions des autorités administratives vont dans le sens d'une interdiction notamment de port de signes d'appartenance religieuse, surtout depuis l'intervention de la loi en 2004 pour les élèves (infra III).

Les solutions relatives aux obligations des personnels ne concernent pas ceux des enseignements supérieurs (cf. concl. Helbronner préc.) sauf s'il y avait excès dans l'utilisation d'une liberté de recherche destinée à garantir les possibilités « de libre développement scientifique créateur et critique» (C. éduc. art. L. 141-6). Mais il a été jugé que l'utilisation dans le service d'une connexion internet de l'établissement pour entrer en communication avec des correspondants en tant qu'adhérent d'une secte est une faute grave (TA Paris, 25 mars 1999, req. $\mathrm{n}^{\circ} 9616402$ / 5,0CAA Paris, 24 janvier 2002, req. $\mathrm{n}^{\circ} 99$ PA 03 034,0).

Trois observations se justifient quant à la situation actuelle de la laïcité. La première est pour souligner que dans l'ensemble le contentieux est mince quant aux obligations de personnels par rapport à ce que l'on peut savoir des difficultés réelles dans les établissements et du nombre réel des contestations de comportements par les parents et... les élèves. Beaucoup de difficultés sont traitées au

11. Nous ne retenons pas ici les problèmes qui à la fin du $\mathrm{XIX}^{\mathrm{e}}$ siècle et au début du $\mathrm{Xx}^{\mathrm{e}}$ siècle ont fait couler beaucoup d'encre sur la conciliation des libertés individuelles et d'obligations hors du service : encadrement de colonies de vacances confessionnelles, décoration pour une procession, enfants scolarisés dans des écoles privées...

12. L'insistance mise par la CEDH de Strasbourg dans un arrêt concernant une institutrice suisse sur cette justification au regard de la CEDH sur le très jeune âge des élèves pourrait conduire à s'interroger sur la compatibilité de nos règles qui ne distinguent pas l'élève de grande section d'école maternelle de l'élève de terminale du lycée pour fixer les interdits imposés au personnel avec nos obligations internationales. 
niveau des établissements. La seconde est pour souligner le risque de voir le contentieux tant des programmes que de la façon dont ils sont enseignés se développer avec l'introduction d'un enseignement sur les religions. La troisième est pour souligner le fait qu'élèves du secondaire et étudiants du supérieur ont souvent dans le cadre des libertés qui leur sont respectivement reconnues des comportements identiques : contestations de la compétence de l'enseignant pour aborder telle question, contestation des propos, exigences de prise en compte d'obligations religieuses... (infra III $)^{13}$.

En 1972 le ministre a consulté le Conseil d'État sur le bien-fondé de l'exclusion d'un enseignant titulaire, donc fonctionnaire, accédant à la prêtrise durant une mise en disponibilité. Le Conseil d'État a estimé que l'exclusion ne serait pas légale en l'absence de disposition législative (CE, AG, avis $\mathrm{n}^{\circ}$ 309.354, 21 septembre 1972, texte cité en annexe aux «Réflexions sur la laïcité », in CE, Études et documents, 2004). La question a été posée à l'administration plus récemment pour l'accès au diaconat. Celle-ci a répondu dans le même sens. Reste... à concilier avec l'obligation de réserve...

\section{B. Le régime des locaux}

Parmi les dispositions concernant les locaux le retrait des crucifix des salles de classe est souvent cité. En réalité, tant pour les locaux d'enseignement que pour les locaux administratifs, il n'y a plus de dispositions législatives spécifiques à l'éducation. La question relève de l'article $28 \mathrm{de}$ la loi du 9 décembre 1905 qui dispose qu' « il est interdit à l'avenir d'élever ou d'apposer aucun signe ou emblème religieux sur les monuments publics ${ }^{14}$. Il n'y a pratiquement pas de contentieux ici. L'arrêt de la Cour administrative d'appel de Nantes du 11 mars 1999 qui juge que le logotype que le département de la Vendée ${ }^{15}$ place sur le fronton des collèges publics «n'a pas été conçu dans un but de manifestation religieuse et ne tend pas à la promotion d'une religion même si une partie de sa composition représente un motif religieux, qu'il ne méconnaît pas l'interdiction de la loi » est une curiosité. S'agissant des locaux utilisés par le service deux questions se posent en pratique. L'une porte sur le recours à des locaux privés d'établissements confessionnels sur décision rectorale pour organiser des examens publics. Cette utilisation est souvent contestée au nom du principe de laïcité et de la neutralité des personnels par les enseignants. Cette contestation a été rejetée au contentieux (TA Bordeaux, 4 mai 2005, fédération syndicale unitaire éducation-enseignement de Lotet-Garonne c. recteur de l'académie de Bordeaux: organisation du baccalauréat). L'autre question est celle de l'utilisation des locaux scolaires par les maires (C. éduc. art. L. 212-15). Cette utilisation doit respecter le principe de neutralité, politique et religieuse, sauf disposition législative contraire, par exemple pour les élections ${ }^{16}$.

\section{Laïcité et droits et obligations des usagers}

Analysées en termes d'obligation incombant au service et à ses personnels certaines dispositions qui ont été présentées dans leurs applications pourraient être reprises en les retenant comme droits au profit des parents ou des élèves. Dans l'ensemble, nous l'avons vu, les solutions du droit sont ici classiques, anciennes, éprouvées, même si nombre de situations nouvelles existent. Il en va différemment avec deux comportements nouveaux et appuyés des usagers, élèves et étudiants, comportements faits d'exigences religieuses et d'interprétations de leurs droits, notamment à certaines libertés, même si les régimes en sont différents pour les premiers et les seconds. Le législateur, l'administrateur, le juge enfin ont été confrontés aux problèmes liés à des refus de respecter des obligations imposées par le service et par des volontés d'expression d'appartenances religieuses.

\section{A. Absences pour motifs religieux}

Aboutissement dans le domaine scolaire d'un accroissement des exigences de lutte contre l'absentéisme scolaire l'article 10 de la loi du 10 juillet 1989 en son premier alinéa dispose que «les obligations des élèves consistent dans l'accomplissement des tâches inhérentes à leurs études » et «incluent l'assiduité» (C. éduc. art. L. 511-1). La loi a été complétée par un décret du 18 février 1991 attaqué au contentieux pour atteinte au libre exercice de la religion juive en imposant l'assiduité le samedi. Le recours en annulation a été rejeté (CE, 14 avril 1995, consistoire central des israélites de France: D., 1995, inf. rap. p. 138 ; JCP, 1995.II. 22437 note Nguyen van Tuong; AJDA, 1995, p. 572). Mais le juge n'écarte pas des dérogations laissant à l'établissement la détermination. Le Ministère insiste sur le souci que doit avoir le chef d'établissement de préserver une scolarité normale (MEN, Direction des affaires juridiques, décembre 2000, Cahiers de l'éducation, janvier-février 2001, p. 29). Les autorisations d'absence ne peuvent avoir un caractère permanent, annuel (TA Lyon, 13 mai 2003, $\mathrm{M}^{\mathrm{me}} \mathrm{S}$. c. recteur de l'académie de Lyon). Elles doivent être ponctuelles. Elles doivent être compatibles avec l'accomplissement des tâches inhérentes aux études en fonction des exigences de ce niveau (CE, 14 avril 1995, Koen : D., 1995,

13. Voir P. Mestre, guide Laïcité et enseignement supérieur (en ligne sur www. cpu. fr), rendu public par la conférence des présidents d'université, et «L’université est un lieu d'enseignement, de débat mais pas de prière ", questions à P. Mestre, AJDA, 2004, p. 1676.

14. Y. Desmurs-Moscet, «Régime des cultes», J. Cl. adm, fasc. 215. Voir notre ouvrage, Le Droit de l'éducation, $2^{e}$ éd., Paris, Hachette éducation, 1998 , p. 241 sq.

15. Deux cœurs entrelacés surmontés d'une croix.

16. Interdiction à un maire de mettre des locaux scolaires à disposition d'un parti politique par convention pour la tenue d'une université d'État : TA Nantes, 29 janvier 1992, Préfet du Maine-et-Loire, Rev. Savoir, 1992, p. 324). 
JP, p. 481, note G. Koubi ; RFDA, 1995, p. 590 concl. Aguila; AJDA, 1995 p. 572, chron. Stahl et Chauveaux - TA Lyon, 13 mai 2003, préc.). Le refus d'accepter le règlement intérieur parce qu'il impose l'assiduité justifie le refus d'inscription de l'élève (CE, 14 avril 1995, Koen, préc.). Mais un refus d'octroi de dérogation est illégalement motivé si le chef d'établissement se fonde sur la seule « impossibilité de déroger au règlement intérieur pour des motifs religieux » sans mentionner l'incompatibilité avec le bon déroulement des études (TA Lyon, 13 mai 2003, $\mathrm{M}^{\text {me }}$ S. préc.). La pression des familles reste vive pour le respect du Sabbat. Elle existe aussi en enseignement supérieur. Les présidents d'université sont confrontés à des demandes d'adaptation d'horaire des cours et examens en fonction des fêtes religieuses. Le guide de la conférence des présidents d'université conseille ici la fermeté.

Dans les enseignements scolaires le refus de participer à certains cours pour des raisons religieuses, par exemple aux cours d'éducation physique et sportive pour des élèves porteuses du foulard islamique, justifie la sanction disciplinaire d'exclusion (CE, 10 mars 1995, M. et $\mathrm{M}^{\text {me }}$ Aoukili - TA Dijon, 6 février 1996, M ${ }^{\text {lles }}$ Matallah et Monadir - CE, 27 novembre 1996, M. et $\mathrm{M}^{\mathrm{me}}$ Jeouit - CE, 27 novembre 1996 , M. et $\mathrm{M}^{\mathrm{me}} \mathrm{Ntar}-\mathrm{CE}, 27$ novembre 1996 , M. et $\mathrm{M}^{\mathrm{me}}$ Wissadane - CE, 27 novembre 1996, M. et $\mathrm{M}^{\mathrm{me}}$ Naderan CE, 20 octobre 1999, Ministre de l'Éducation nationale c. époux Aït Ahmad: JCP, 200o.II.10306 note G. Guclielmi et G. Koubi ; AJDA, 2000, p. 165 note F. de la Morena - CAA Lyon, 15 juillet 1999, Ministre de l'Éducation nationale c. Mahamood: Juris-data $\mathrm{n}^{\circ} 107973$ - TA Caen, 5 octobre 1999, Dogru : Juris-data n ${ }^{\circ} 105342$ - TA Caen, 5 octobre 1999, Kervanci : Juris-data $\mathrm{n}^{\circ}$ 104283). L'exclusion ne peut être prononcée par le seul chef d'établissement, elle ne peut résulter que d'une procédure disciplinaire (TA CergyPontoise, ord., 21 octobre 2004, req. $\mathrm{n}^{\mathrm{o}}$ 0407980, M. Bikranjit Singh: AJDA, 2004, p. 2023 : concerne un refus d'ôter le turban des sikhs).

Chaque année le bulletin officiel de l'Éducation nationale publie une liste des jours des fêtes religieuses des diverses confessions durant lesquels les absences des élèves sont autorisées.

\section{B. Manifestations d'appartenance}

Il s'agit ici, pour l'essentiel, du port de signes manifestant une appartenance religieuse. Mais les manifestations de convictions ne se limitent pas à cela. Tout le service public est concerné par des comportements religieux qui mettent en cause la laïcité, ou peuvent le faire. Certains écrits ont signalé l'attitude d'élèves de l'enseignement secondaire ou d'étudiants contestant des propos professoraux au nom de telle ou telle doctrine religieuse, ou le droit pour une enseignante d'évoquer telle ou telle question. Le fait que des garçons aient psalmodié des versets du Coran dans des couloirs de lycées - non sanctionné disciplinairement d'ailleurs - a été relevé comme l'ont été l'utilisation religieuse de locaux affectés à des associations étudiantes ou le détournement d'autorisation de locaux. La circulaire du 18 mai $2004{ }^{17}$ relative à la mise en œuvre de la loi $\mathrm{n}^{\circ}$ 2004-228 du 15 mars 2004 porte d'une certaine manière témoignage de l'existence de tels faits et entend y faire obstacle ${ }^{18}$.

L'enseignement supérieur n'est pas concerné par le problème principal qui est le port de signes d'appartenance religieuse. Le juge en a ainsi décidé (CE, 26 juillet 1996, Université de Lille II, Rec. tables p. 924). La loi $\mathrm{n}^{\mathrm{o}}$ 2004-228 du 15 mars 2004 encadrant en application du principe de laïcité le port de signes ou tenues manifestant une appartenance religieuse ne concerne pas l'enseignement supérieur mais les seuls écoles, collèges et lycées ${ }^{19}$.

Ce que l'on peut désormais appeler l'histoire du problème du port des signes d'appartenance religieuse, reprise ici à grands traits, débute en 1989. Saisi par le ministre de l'Éducation nationale le Conseil d'État rend le 27 novembre 1989 un avis sur la compatibilité entre le principe de laïcité et le port de signes d'appartenance religieuse ${ }^{20}$. Celui-ci est suivi par le ministre en une circulaire du 12 décembre $1989^{21}$. Le port des signes n'est pas contraire au principe de laïcité. Il s'inscrit dans le cadre de la liberté d'expression reconnue aux élèves par le second alinéa de l'article 10 de la loi $\mathrm{n}^{\circ} 89-486$ du 10 juillet $1989^{22}$. Mais l'exercice de ce droit est encadré par des limites précises: pas de prosélytisme, de propagande qui porteraient atteinte à la liberté des autres élèves, pas d'atteinte à la santé et à la sécurité ${ }^{23}$, pas de perturbation des activités d'enseignement ou de l'ordre dans l'établissement. C'est ce que précise l'article $1^{\text {er }}$ du décret d'application de la loi (C. éduc. art. L. 511-2), le décret $\mathrm{n}^{\circ}$ 91-173 du 18 février $1991^{24}$. En nombre et par vagues des recours sont formés contre des décisions d'exclusion. Celles-ci sont prises au terme de procédures jugées délicates de mise en œuvre. La difficulté d'interprétation du droit est souvent évoquée

17. JORF, 22 mai 2004, p. 9 033. Voir O. Dord, «Laïcité à l'école : l'obscure clarté de la “circulaire Fillon” du 18 mai 2004", AJDA, 2004, p. 1523.

18. Extraits: «[...] les convictions religieuses des élèves ne leur donnent pas le droit de s'opposer à un enseignement... [de] contester le droit d'un professeur; parce que un homme ou une femme d'enseigner certaines matières ou le droit d'une personne n'appartenant pas à leur confession de faire une présentation de tel ou tel fait historique ou religieux» $\left(\mathrm{II}-2.4, \S_{3}\right)$.

19. JORF, 17 mars 2004. Voir notre commentaire «La loi sur la laïcité, une volonté politique au centre de débats de société», AJDA, 2004, p. 704.

20. J. Rivero, «Laïcité scolaire et signes d'appartenance religieuse», RFDA, 1990 p. 1 sq.; J.-P. C., «Le principe de liberté et les signes d'appartenance religieuse ", AJDA, 1990, p. 39 .

21. Voir notre commentaire «La circulaire Jospin du 12 décembre 1989 », RFDA, 1990, p. 10.

22. Texte rendu responsable par certains des situations créées par le port du foulard islamique. Ce texte introduit en droit interne une liberté reconnue par des dispositions internationales ratifiées par la France. L'avis du Conseil d'État énumère les textes applicables dont ceux qui comportent ces dispositions.

23. Il s'agit des limites même que prévoit l'article 9-2 de la Convention européenne de sauvegarde des Droits de l'homme et des libertés fondamentales au droit de manifester sa religion ou ses convictions.

24. Voir notre commentaire « Droits et obligations des élèves du second degré », AJDA, 1991, p. 366. 
et contestée. La jurisprudence est fournie ${ }^{25}$. Dès 1995 dans ses conclusions dans l'affaire association un Sisyphe le commissaire du gouvernement R. Schwartz indiquait que ce qu'il qualifiait de jurisprudence de conciliation n'avait pas toujours localement permis de régler les conflits (CE, 10 juillet 1995, association un Sisyphe : AJDA, 1995 p. 644 concl. R. Schwartz; JCP, 1995.II.22519, note A. Asworth). C'est dans ce contexte que la commission Debré de l'Assemblé nationale puis la commission Stasi se saisissent de la question. La loi n ${ }^{\circ}$ 2004-228 du 15 mars 2004 est votée à une écrasante majorité dans les deux chambres du Parlement (réf. préc.). Elle est complétée par une circulaire, précitée, du 18 mai 2004. La loi s'applique à Mayotte et aux seuls établissements relevant de l'État en NouvelleCalédonie et aux îles Wallis et Futuna. Elle s'applique aux écoles, collèges et lycées publics. Les signes et tenues sont interdits lorsque leur port conduit immédiatement à faire reconnaître une appartenance religieuse : voile islamique, quelque soit le nom qu'on lui donne, kippa, croix de dimension manifestement excessive. La loi ne remet pas en cause le droit de porter des signes discrets. L'exclusion définitive a été prononcée en application de la loi dans quarante sept cas. Dans vingt cinq cas les tribunaux administratifs ont été saisis de requête en annulation. Le bilan de la jurisprudence ne peut encore être établi. Mais hors problèmes de procédure, au fond, certaines décisions d'exclusion ont été confirmées (TA Caen, 7 juin 2005, M. et $\mathrm{M}^{\mathrm{me}}$ Kervance). Une décision de justice marque nettement que ce n'est pas le signe qui est répréhensible en lui-même mais le fait qu'il soit porté de manière à manifester ostensiblement l'appartenance religieuse (TA Caen, 7 juin 2005, préc., port en permanence d'un bonnet noir substitué au «voile»).

Les refus de respecter l'obligation d'assiduité, les exigences quant aux fixations de dates d'examens, la contestation d'enseignements et d'enseignants, le port de signes d'appartenance sont autant de manifestations individuelles que leurs auteurs entendent fonder sur une interprétation des libertés de conscience et d'expression, interprétation que refusent au nom de la laïcité les textes, l'administration, le juge. Mais il existe, tant en enseignement scolaire qu'en enseignement supérieur, des situations dans lesquelles la revendication porte sur l'exercice collectif de libertés publiques reconnues.

\section{Libertés publiques des élèves et étudiants et manifestations religieuses}

Dans les collèges et les lycées les élèves disposent de la liberté d'information et de la liberté d'expression (C. éduc. art. L. 511-2). La loi et son décret d'application $\mathrm{n}^{\circ}$ 91173 du 18 février 1991 précisent que l'exercice de ces droits ne peut porter atteinte à la liberté et aux autres droits des autres membres de la communauté éducative, ni au pluralisme et au principe de neutralité. Les chefs d'établissement ont donc le pouvoir d'interdire, par exemple, certaines réunions organisées par les élèves avec des intervenants dont les propos offenseraient les consciences dans le domaine religieux. Ils pourraient interdire la publication d'articles dans les journaux lycéens.

La loi précise que les usagers du service public en enseignement supérieur « disposent de la liberté d'information et d'expression à l'égard des problèmes politiques, économiques, sociaux et culturels » et qu' «ils exercent cette liberté à titre individuel et collectif, dans des conditions qui ne portent pas atteinte aux activités d'enseignement et de recherche et qui ne troublent pas l'ordre public» (C. éduc. art. L. 811-1). Dans une réponse à un établissement la Direction des affaires juridiques du Ministère (Lettre $\mathrm{DAJ} \mathrm{B}_{1} \mathrm{n}^{\circ}$ 03-233 du 2 juillet 2003 publiée dans la lettre d'information juridique du Ministère $\mathrm{n}^{\circ} 78$ d'octobre 2003, p. 32) énumère un certain nombre de limites à la liberté des étudiants ${ }^{26}$ : prosélytisme et pression sur d'autres étudiants pour qu'ils portent dans l'établissement des signes d'appartenance religieuse (CE, 2 avril 1997, Ministre de l'Éducation nationale c. époux Mehila, req. $\mathrm{n}^{\circ} 173103$ ), provocation de mouvements de protestation causant un trouble au fonctionnement de l'établissement ou au déroulement des enseignements (CE, 10 mars 1995, époux Aoukili: Rec. p. 122 - CE, 27 mars 1996, Ligue islamique du Nord, M. et $\mathrm{M}^{\mathrm{me}}$ Chabou et autres: Rec. p. 461), refus d'accès aux réunions qu'ils organisent dans l'établissement opposé à des usagers sur le fondement de discrimination (sexuelle, religieuse), remise en cause de la mixité des enseignements, mise en cause de la santé, de l'hygiène et de la sécurité, atteinte à la dignité des personnes... La lettre préconise d'inclure dans le règlement intérieur de l'établissement des dispositions aménageant la liberté d'expression des usagers au regard du respect du principe de neutralité. Elle estime que «les étudiants étant, par rapport au service public de l'enseignement supérieur dans une situation réglementaire, la mise en place d'une charte... ne saurait établir une relation de nature contractuelle engageant le signataire vis-à-vis de l'établissement ».

\section{Laïcité et obligation hors service public. L'imposition par voie contractuelle d'obligations de laïcité}

Une situation retient l'attention par rapport à un schéma classique d'opposition entre un service public soumis au principe d'égalité d'accès et à une règle de neutralité de son organisation, de ses enseignements et de ses moyens et les établissements privés d'enseignement, intervenant dans le cadre de la liberté de l'enseignement, principe fondamental reconnu par les lois de la République (CC 23 novembre 1977, $\mathrm{n}^{\circ}$ 77-87 DC). Il s'agit de l'encadrement de certaines décisions de certains de ces derniers.

25. Les arrêts du Conseil d'État et les principales décisions des Tribunaux administratifs avec les notes et commentaires peuvent être trouvés sous l'article L. 511-2 du Code de l'éducation dans l'édition précitée.

26. Elle le fait parfois... en s'appuyant sur une jurisprudence qui concerne l'enseignement secondaire du second degré. 
Dans le service public l'accès ne saurait être refusé à un élève pour motif religieux tant durant la période de scolarité obligatoire que pour la poursuite facultative d'études. La non-discrimination est ici simple application du principe de neutralité des services publics conséquence, ellemême, du principe d'égalité. L'article L. 141-1 du Code de l'éducation le précise «comme il est dit au treizième alinéa du Préambule de la Constitution du 27 octobre 1946 confirmé par celui de la Constitution du 4 octobre 1958 la Nation garantit l'égal accès de l'enfant... à l'instruction». En principe chaque établissement privé peut accueillir les élèves qu'il veut bien inscrire, la seule limite étant de ne pas pratiquer une discrimination sur le fondement de l'origine, donc de la race ou de l'ethnie, condamnable sur la base de l'article.225-1 du Code pénal. La sélection sur le fondement de critères religieux est d'autant plus concevable que les établissements privés sont pratiquement tous confessionnels et que la loi reconnaît leur caractère propre $^{27}$.

Telle n'est pas la situation de la grande majorité des établissements. L'article $1^{\text {er }}$, alinéa 4 de la loi Debré du 31 décembre 1959 dispose que dans les établissements qui ont passé avec l'État un contrat « tous les enfants sans distinction d'origine, d'opinion ou de croyance ont accès ». Codifié, le texte est l'article L. 442-1 du Code de l'éducation qui est placé en tête des développements consacrés au contrôle de l'État sur les établissements d'enseignement privés. Le texte est-il respecté? Certainement pour nombre d'établissements catholiques dont on sait qu'ils accueillent des non-catholiques et que, par exemple, ils On ne peut exclure que certains établissements de tendance «intégriste » ne sélectionnent en fait sur le critère des croyances. Mais aucune procédure de testing n'a été utilisée et les contrats d'association avec l'État n'en sont nullement affectés. Il existe une forte présomption que certains des établissements juifs sous contrat d'association sélectionnent leurs élèves ou s'il n'y a pas « sélection active» que ces établissements ne voient venir à eux que certains élèves ce qui est d'autant plus vraisemblable que certains ne respectent pas les programmes, ce à quoi ils sont légalement tenus ${ }^{28}$.

La loi n'impose pas seulement le respect des programmes. La loi de 1959 précise que «l'établissement, tout en conservant son caractère propre, doit donner cet enseignement dans le respect total de la liberté de conscience » (C. éduc. art. L. 442-1) ${ }^{29}$. L'enseignement religieux peut être dispensé dans les écoles et établissements privés mais la loi prévoit que «l'enseignement religieux est facultatif dans les écoles privées» (C. éduc. art. L. 141-3).

Pour le Conseil d'État le respect du caractère propre de l'établissement ne doit pas porter atteinte à la liberté de conscience des personnels dont les obligations doivent s'apprécier en prenant en considération les fonctions exercées (CE, 20 juillet 1990, Association familiale de l'externat St Joseph c. Vivien et autres).

$$
\star *
$$

Les développements précédents ont retenu les divers aspects de la place et du rôle de la laïcité dans le service public de l'éducation et dans les établissements privés qui lui sont associés. Ceci n'épuise pas totalement la question, plus large, de la prise en compte du fait religieux par le droit de l'éducation. Une solution mérite à ce titre d'être évoquée.

Aux termes de l'article L. 131-2 du Code de l'éducation l'obligation d'instruction peut être satisfaite soit par la scolarisation dans un établissement public ou privé soit «dans les familles par les parents ou l'un d'entre eux ou toute personne de leur choix». L'option pour tel ou tel mode d'instruction peut, à l'évidence, reposer sur une motivation religieuse. Les écoles - mot entendu ici lato sensu - publiques accueillent sans discrimination des élèves de milieux catholiques, protestants de différentes confessions, juifs, musulmans, boudhistes, orthodoxes, agnostiques, athées. Les écoles confessionnelles quand elles existent peuvent recevoir les élèves de leur confession - et pas uniquement, nous l'avons vu, quand elles sont liées à l'État par contrat. L'éducation dite familiale était et reste rare, limitée à des milieux sociaux favorisés, à des opposants de principe à l'institution scolaire, à des milieux traditionalistes... Mais elle avait tendance à se développer. Il a été constaté que certaines familles faisaient usage du droit de choisir le mode d'instruction pour confier leurs enfants à des sectes. Le législateur a réagi. Le contrôle public a été renforcé en 1998 sur les motivations avancées pour choisir d'instruire l'enfant en milieu familial et sur le contenu effectif de la formation dispensée (C. éduc. art. L. 131-10). La loi a été complétée par un décret $\mathrm{n}^{\circ}$ 99-224 du 23 mars 1999 relatif au contenu des connaissances des enfants instruits dans la famille ou dans des établissements d'enseignement privés hors contrat ${ }^{30}$ et une circulaire $\mathrm{n}^{\circ}$ 99-070 du 14 mai 1999 relative au renforcement du contrôle de l'obligation scolaire ${ }^{31}$.

27. Ces établissements sont quasiment tous catholiques. Il n'existe que très peu d'établissements protestants et moins encore musulmans : sur la situation, voir B. Toulemonde, «La loi Debré du 31 décembre 1959: une construction juridique originale », in La Loi Debré. Paradoxes de l'État éducateur? (Actes du colloque d'Amiens, 9-10 décembre 1999), Amiens, CRDP de l'académie d'Amiens (Documents, actes et rapports pour l'éducation), 2001 , p. 109.

28. D. Borne, doyen de l'Inspection générale de l'Éducation nationale, co-président du Comité national de réflexion et de proposition sur la laïcité à l'école : « [...] il n'y a pas eu d'enquête quant à l'enseignement donné dans les écoles privées sous contrat juives. Je m'exprime avec précaution. Néanmoins, on assiste, dans l'enseignement même de ces écoles, à une tendance à des formes de communautarisme. Quelques rapports d'inspecteurs venus inspecter les cours d'histoire m'ont indiqué que les programmes d'histoire ne sont pas toujours suivis, alors que cela devrait être le cas. Je ne voudrais pas que cette situation soit généralisée à toutes les écoles juives sous contrat, mais il est certain que dans quelque cas, c'est patent et clair » (cité in $L a$ Laïcité à l'école: un principe républicain à réaffirmer, Assemblée nationale, rapport $\mathrm{n}^{\circ}{ }_{1275}$, documents d'information, décembre 2003 , t. $2, \mathrm{p} .78$ ).

29. Sur le caractère propre et les obligations au titre de la laïcité : B. Toulemonde, art. préc. note 27 ; N. Ach, La Liberté de l'enseignement face à l'intervention publique, th. Metz, 2004, ronéotypée, p. 335 sq.

30. JORF, 24 mars 1999. Ce décret est codifié : C. éduc. art. D.131-11 à D.131-17.

31. Voir Quinzaine juridique, 14 juin 1999, $\mathrm{n}^{\circ} 162$, p. 15. 